\title{
As interfaces do espaço relacional de idosas cuidadoras ${ }^{1}$
}

\author{
Alessandra Vieira de Almeida² \\ Simone Caldas Tavares Mafra ${ }^{3}$ \\ Emília Pio Silva ${ }^{4}$ \\ Solange Kanso ${ }^{5}$ \\ Sheila Maria Doula ${ }^{6}$
}

\section{Resumo}

Buscou-se examinar o espaço relacional das idosas cuidadoras, investigando as consequências do ato de cuidar no cotidiano das mesmas e suas redes de apoio institucionais e familiares. Entrevistaram-se 24 cuidadoras entre março e abril de 2017, utilizando-se a técnica SnowBall para a seleção da amostra. Realizou-se a Análise Textual, apoiada pelo software IRaMuTeQ. Os resultados revelaram que o espaço relacional da pessoa dependente e da idosa cuidadora é marcado por consequências positivas e negativas, advindos da prestação de cuidados. Além disso, observou-se a predominância da rede de apoio informal e a importância desta para melhor desempenho da tarefa de cuidar.

\section{Palavras-chave}

Envelhecimento; Mulheres; Relação de Cuidado.

\section{Interfaces of the relational space of elderly caregivers}

\begin{abstract}
We sought to examine the relational space of elderly caregivers, investigating the consequences of the act of caring on their daily lives and their institutional and family support networks. 24 caregivers were interviewed between March and April 2017, using the SnowBall technique for sample selection. Textual Analysis was carried out, supported by the IRaMuTeQ software. The results revealed that the relational space of the dependent person and the elderly caregiver is marked by positive and negative consequences, arising from the provision of care. In addition, there was a predominance of the informal support network and its importance for better performance of the care task.
\end{abstract}

\section{Keywords}

Aging; Women; Care relationship.

Artigo recebido: novembro de 2020

Artigo aceito: dezembro de 2020 


\section{Introdução}

Ao chegar na etapa da vida denominada velhice, as pessoas geralmente apresentam uma condição de multimorbidade, ou seja, são acometidas por duas ou mais doenças crônicas, sendo estas doenças persistentes e exigentes de cuidados permanentes. Pode-se dizer que, em função do aumento da expectativa de vida, as pessoas viverão mais, contudo, estarão sujeitas a enfrentarem as doenças crônicas que levam a perda da autonomia, da capacidade funcional e principalmente da independência.

A partir disso, a pessoa idosa passa a demandar da ajuda de terceiros para a realização de suas atividades de vida diária (AVD`s), como comer, tomar banho, ir ao banheiro, vestir-se, andar em casa de um cômodo para o outro no mesmo andar e deitar-se, e também para as atividades instrumentais básicas (AIB`s), entendidas como fazer compras, cuidar do próprio dinheiro, administrar medicamentos e utilizar meios de transporte. Então, surge a figura do cuidador e, em torno dele, inicia-se a construção de uma rede de apoio. Para Biolo e Portela (2010), a rede de apoio pode ser entendida como suporte funcional, econômico e/ou afetivo oferecido por outros membros da família, dos vizinhos, de grupos da comunidade, chamada rede de apoio informal, ou até mesmo, o apoio social de instituições e profıssionais, denominada rede de apoio formal, essenciais para amenizar a sobrecarga de quem exerce a tarefa de cuidar.

Em se tratando da mulher idosa como a principal cuidadora, acredita-se que a rede de apoio se torna ainda mais importante, tendo em vista as necessidades e dificuldades trazidas pela velhice. Além disso, a idosa deixa de cuidar da sua própria saúde para cuidar do idoso dependente. O estudo de Aerosa (2014) evidenciou que o papel de cuidar acarreta consequências que se caracterizam tanto de forma negativa como positiva e, Guedes (2011) reforça que a sobrecarga é uma das principais consequências negativas para o cotidiano do cuidador. Mayor (2014) corrobora com essa discussão ao afırmar que os aspectos positivos do ato de cuidar envolvem a sensação de dever cumpri- 
do e realização pessoal. No entanto, prevalecem os fatores negativos como a sobrecarga, a insegurança e os problemas familiares, sendo que esses podem influenciar diretamente na saúde dos cuidadores e das pessoas demandantes de cuidado.

Diante dessa discussão, o presente estudo teve como objetivo examinar o espaço relacional das idosas cuidadoras, investigando as consequências do ato de cuidar no cotidiano da mesma e suas redes de apoio institucionais e familiares, tendo em vista que a relação de cuidado pode estar associada a uma reestruturação da família diante das demandas do(a) idoso(a) dependente.

\section{Métodos}

Trata-se de um estudo de caso, com caráter qualitativo de investigação e abordagem exploratória e descritiva, realizado com 24 mulheres idosas cuidadoras de pessoas idosas debilitadas, física e/ou mentalmente, no município de Viçosa, MG. Foram incluídas na pesquisa apenas idosas que possuíam vínculo familiar com a pessoa demandante de cuidado. Destaca-se que a população idosa viçosense possui maior número de mulheres, cuja porcentagem foi de 6,1\%, com um diferencial de $1,2 \%$ a mais do que os homens idosos no município $(4,9 \%)$, como exposto pelo IBCE (2010). A estimativa da população para o ano de $2015^{7}$ apontou uma variação nestas proporções, revelando o crescimento contínuo desse contingente populacional, em que a população idosa correspondeu a $12,8 \%$ da população total do município, com $11,9 \%$ de idosos e $13,6 \%$ de mulheres idosas, mantendo-se como a maioria entre a população idosa (MINISTÉRIO DA SAÚDE/DATASUS, 2015). Por meio dos dados apresentados, verifica-se que a referida cidade é propícia para os estudos em envelhecimento.

O procedimento para a coleta de dados foi realizado da seguinte maneira: buscou-se na rede de contatos da pesquisadora uma idosa cuidadora. A partir dessa, foi sendo feita uma rede de contatos, em que cada cuidadora entrevistada indicava outra de seu convívio na mesma 
condição, formando um grupo a ser abordado, até que a pesquisa alcançasse o seu objetivo e o seu ponto de saturação. Esse método da Bola de Neve, também conhecido como cadeia de informantes, segundo Biernacki e Waldorf (1981), serviu de apoio para a execução da técnica de coleta de dados principal deste estudo, a entrevista semiestruturada (Apêndice). A construção do roteiro de entrevista baseou-se em Miranda (2014), Andrade (2009) e Mazza (2008), cujos estudos abordaram o cuidado em contexto domiciliar, a qualidade de vida dos idosos e o cuidado sob o olhar do idoso, respectivamente. Deste modo, as cuidadoras que aceitaram participar da pesquisa foram visitadas em suas respectivas residências, com dia e horário marcados, segundo a possibilidade das mesmas, onde procedeu-se a execução da pesquisa. O período entre março e abril de 2017 compreendeu a coleta de dados.

O material transcrito referente ao conteúdo das entrevistas foi analisado através do software IRaMuTeQ (Interface de R por análise Multidimensional de Texto e Questionário), de origem francesa, criado por Pierre Ratinaud. O software oferece cinco tipos de testes na parte textual: estatísticas gerais, especificidades, classificação hierárquica descendente (CHD), similitude e nuvem de palavra. (CARMARCOS E JUSTOS, 2013).

Antes de iniciar os testes, cada uma das entrevistas foi agrupada em arquivo único, denominado corpus de análise. Para a análise dos resultados desse artigo optou-se pela CHD, análise de similitude e nuvem de palavras, buscando verificar as principais temáticas apontadas nas entrevistas com as mulheres cuidadoras. De acordo com Camargo e Justo (2013), a CHD apresenta um conjunto de palavras estatisticamente significativas, contribuindo para a realização de uma análise mais qualitativa dos resultados. Já a análise de similitude permite identificar as coocorrências entre as palavras, indicando a relação entre as mesmas. E a nuvem de palavras é uma análise lexical mais simples e possibilita uma rápida identificação das palavras-chave de um corpus. Ela visa agrupar e organizar as palavras de modo gráfico em função da sua frequência. 
A pesquisa foi aprovada pelo Comitê de Ética em Pesquisa (CEP) da Universidade Federal de Viçosa - UFV, cujo parecer corresponde ao número 1.956.311, enviado em 09 de março de 2017. Em todo o processo da pesquisa foram atendidos os princípios éticos dispostos na resolução N 466/2012 do Conselho Nacional de Saúde.

\section{Resultados e discussão}

Os resultados referentes ao espaço relacional das idosas foram processados pelo software IRaMuTeQ. Nesse, a análise da Classificação Hierárquica Descendente (CHD) possibilitou o agrupamento de temáticas a partir das respostas das cuidadoras entrevistadas. O conteúdo textual resultou em 101 Segmentos de Texto (ST), com 588 palavras diferentes. As palavras e os ST se dividiram em quatro classes temáticas, conforme o dendrograma apresentado na Figura 1. 
Figura 1 - Dendrograma da análise Classificatória Hierárquica Descendente

\section{CORPUS}

CLASSE 2

Rede de apoio familiar nas relações de cuidado 20 STs $19,8 \%$

\begin{tabular}{|l|c|c|}
\hline PALAVRAS & $\mathrm{N}^{\circ}$ & $\mathrm{x}^{2}$ \\
\hline $\begin{array}{l}\text { Tipos de } \\
\text { ajuda }\end{array}$ & $\mathbf{1 8}$ & $\mathbf{8 8 . 7 1}$ \\
\hline
\end{tabular}

\begin{tabular}{|l|l|l|}
\hline Tarefa & 17 & 82.78 \\
\hline
\end{tabular}

\begin{tabular}{|l|l|l|}
\hline Doméstico & 15 & 71.35 \\
\hline
\end{tabular}

\begin{tabular}{l|l|l|}
\hline Companhia & 16 & 65.46 \\
\hline
\end{tabular}

\begin{tabular}{|l|l|l} 
Rede de & 21 & 53.08
\end{tabular}
apoio

\begin{tabular}{|l|l|l|}
\hline Cuidado & 8 & 35.19 \\
\hline
\end{tabular}

\begin{tabular}{|l|c|c|}
\hline pessoal & 8 & 35.19 \\
\hline Importante & 6 & 25.83 \\
\hline Neto
\end{tabular}

\begin{tabular}{|l|l|l|}
\hline Neto & 5 & 21.30 \\
\hline
\end{tabular}

\begin{tabular}{|l|l|l|}
\hline Presença & 4 & 16.87 \\
\hline
\end{tabular}

\begin{tabular}{|l|l|l|}
\hline Idoso & 19 & 11.20 \\
\hline
\end{tabular}

\begin{tabular}{|l|c|c|}
\hline Filho & 23 & 10.51 \\
\hline Apoio & 8 & 9.97 \\
\hline
\end{tabular}

\begin{tabular}{l|l|l|}
\hline Compra & $\mathbf{6}$ & $\mathbf{8 . 8 2}$ \\
\hline
\end{tabular}

\begin{tabular}{|l|l|l|}
\hline Financeiro & 4 & 7.99 \\
\hline
\end{tabular}

\begin{tabular}{|l|l|l|}
\hline Transporte & 4 & 7.99 \\
\hline
\end{tabular}

\begin{tabular}{l|l|l|}
\hline Rede & 3 & 4.28
\end{tabular}

\begin{tabular}{|l|c|c|}
\hline Vizinho & 3 & 4.28 \\
\hline Sobrinho & 3 & 4.28 \\
\hline Ajuda & 6 & 3.66 \\
\hline Irmão & 13 & 3.27 \\
\hline Médico & 4 & 2.39 \\
\hline Cuidado & 4 & 2.39 \\
\hline
\end{tabular}

Fonte: Elaboração dos autores com base nos relatórios do software IRaMuTeQ, 2018.

Como pode ser observado na Figura 1, o corpus sofreu duas partições a partir do processamento do programa computacional. Houve a primeira partição, que originou duas aglomerações de classes, a Classe 2, a que se denominou Rede de apoio familiar nas relações de cuidado, sendo desmembrada nas outras três classes, em que constam as Repercussões do cuidado para a mulher idosa (Classes 3 e 4). 
Os tópicos sobre as temáticas referentes a cada classe e seus agrupamentos são apresentados a seguir. Porém, após a leitura e análise dos segmentos de textos da Classe 1, optou-se por retirá-la, uma vez que foi observada uma heterogeneidade em relação às demais classes e seus conteúdos.

\section{A rede de apoio familiar nas relações de cuidado da pessoa idosa: Classe 2}

No estudo em questão, a mulher idosa assumia a figura do cuidador familiar, sendo esse cuidador, geralmente as esposas e/ou filhas. Embora o cuidado seja uma atribuição dessa mulher, observou-se, de forma preponderante, a presença de pessoas do cotidiano, familiares e amigos, colaborando com algumas tarefas, estabelecendo uma rede de apoio informal. Segundo Dá Mesquita (2011) a rede de apoio informal inclui simultaneamente indivíduos (familiares, amigos, vizinhos) e os grupos sociais (clubes, associações, igrejas etc.), que fornecem apoio nas atividades cotidianas.

Os resultados obtidos nesta pesquisa estão de acordo com o conceito de Dá Mesquista (2011) e Guedes (2011), visto que a rede de apoio era formada por filhos, netos, irmãos, sobrinhos e vizinhos, conforme ilustram as falas abaixo. Destacam-se, ainda, os tipos de ajuda que ofereciam, sendo as tarefas domésticas, a companhia, o cuidado pessoal do idoso e a ajuda para compras os mais evidentes. Os estudos de Domingues et. al. (2012) revelaram que a rede de apoio citada pelas idosas são as que mais se mostram presentes e referenciadas pelas pessoas idosas.

"A minha rede de apoio são os filhos e quando preciso sair, ficam com ele. (Ent. 7)

"A minha rede de apoio são minhas filhas e neto." (Ent. 04)

"A minha rede de apoio é os irmãos e sobrinhas." (Ent. 09)

A importância dessa rede de apoio foi comentada pelas idosas, que afırmaram se sentir amparadas em seu cotidiano, conforme ilustram as entrevistas 09 e 13. Com relação à pessoa idosa, Araújo et. al. (2013) 
revelaram que o apoio dos familiares no exercício do ato de cuidar é crucial para os idosos enfrentarem as enfermidades.

(...) é importante a presença da rede de apoio por causa do companheirismo (Ent. 09)

(...) eu acho importante a presença da rede de apoio, porque ela dá apoio e segurança (Ent. 13)

Além dos resultados trazidos pela análise do IRaMuTeQ sobre as redes de apoio informais, dezesseis entrevistadas também relataram receber apoio da prefeitura, tais como o fornecimento de medicamentos, atendimentos com profissionais da saúde (médico, nutricionistas, fisioterapeutas e psicólogos) e as visitas dos agentes de saúde do Programa Saúde da Família. Segundo Souza et al. (2006), a rede de apoio pode ser entendida como um conjunto de conexões ou vínculos significativos e é composta por indivíduos que interagem com a pessoa demandante de cuidado, podendo ser os familiares, os vizinhos, os amigos, os profissionais de saúde, entre outros.

De acordo com Dá Mesquita (2011), parece consensual que os idosos que possuem maior rede social, obtêm maior suporte social e alta-frequência de interações sociais que leva ao menor declínio funcional e à maior longevidade. Em contrapartida, a ausência dessas redes, pode se tornar um fator agravante para o cuidador, pois essa relação implica diretamente na realização do cuidado de si, devido à falta de tempo direcionado à tarefa do cuidado (ARAÚJO, et. al., 2013). Desta forma, ressalta-se que o apoio e a segurança propiciada pela presença das redes de apoio contribuem para a diminuição do peso que a tarefa de cuidar pode trazer para o cotidiano das cuidadoras que repercute em sua saúde, em sua vida social, entre outras dimensões.

\section{As repercussões do cuidado para a mulher idosa}

As consequências do cuidado: Classe 4

O envelhecimento da população idosa traz consigo a necessidade de cuidado, sendo essa uma tarefa árdua para o cuidador. Diante do 
processo de feminização da velhice, esse cuidado tem sido ofertado por mulheres já idosas, que em muitos momentos tem enfrentado dificuldades no exercício do mesmo, sendo essas dificuldades de ordem emocional e/ou psíquica (ALMEIDA, 2015).

Dentro desse contexto, os resultados desta pesquisa revelaram que o cuidado domiciliar ocasiona uma série de consequências positivas e negativas para a cuidadora. Os estudos de Cruz et. al. (2010) e Aerosa (2014), também revelaram esta realidade, em que o cuidado informal traz consequências negativas, mas também se reveste de aspectos positivos. Essas consequências encontram-se ilustradas na Figura 2.

Figura 2. Nuvem de palavra relacionada com as consequências positivas e negativas do ato de cuidar.

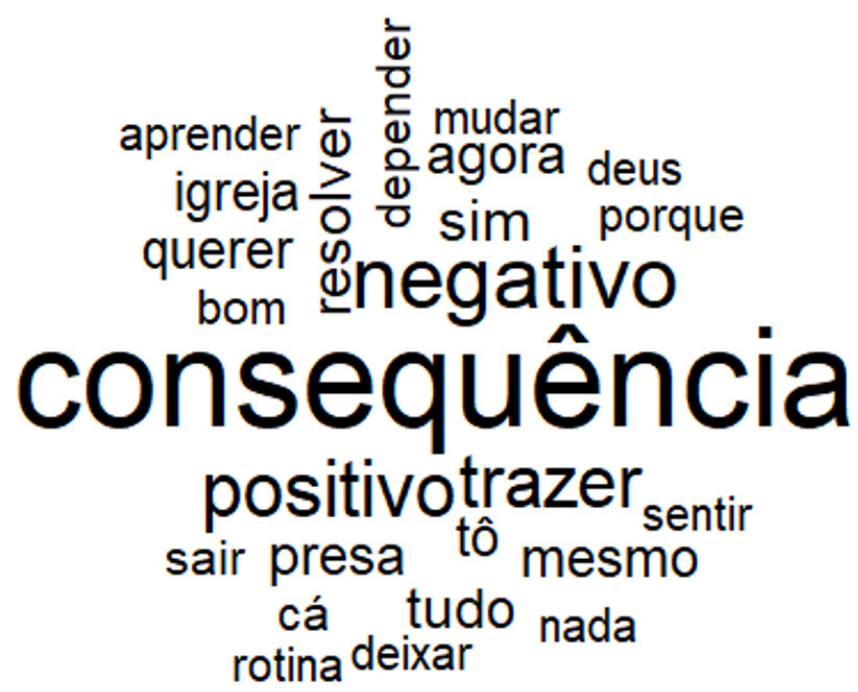

No centro da figura, destaca-se a palavra "consequência" por ser ela o cerne da questão em análise, em seguida as palavras "positivo" e "negativo", que representam os dois lados das consequências do cuidar mencionadas pelas idosas. Em torno dessas, observou-se que as palavras “presa”, “resolver”, “tudo”, “querer”, “igreja”, “depender”, “sair”, "deixar”, “mudou”, “rotina”, "pedir” e "antes” traduzem as consequên- 
cias negativas da tarefa do cuidar, que podem ser confirmadas a seguir pelos trechos das falas das idosas.

"Muita coisa mudou para pior, porque quando ele estava bom, ele fazia tudo, agora é essa luta para lá e pra cá para eu cuidar. A coisa piorou mesmo." (Ent. 1)

"A consequência negativa é a dificuldade para sair. Não posso sair, é difícil! De positivo não tem nada, doente não traz nada positivo não, como é que traz?" (Ent. 7)

"Fiquei mais presa, porque não posso deixá-la sozinha." (Ent. 13)

"Eu deixei muito a minha família em segundo plano, eu acho que foi um erro meu." (Ent. 14)

Assim como nos estudos de Alves e Monteiro (2015), verificou-se que as relações sociais são afetadas e ocorrem mudanças negativas no cotidiano em função do cuidado prestado. Domingues et. al. (2012) afirmaram que o cuidado domiciliar pode trazer o desgaste nas relações e tornar uma obrigação social desprazerosa.

Em relação às consequências positivas, de um modo geral, as entrevistadas reconheceram a tarefa de cuidar como uma dádiva divina, ou seja, algo dado por Deus, sendo o cuidado um ato de amor, retribuição e um grande aprendizado. Na Figura 2, palavras evocadas como “Deus”, “bom”, “nada”, “sentir”, “aprender", “eu” e "mim” explicam os pontos positivos do cuidado. Os trechos abaixo evidenciam a presença dessas palavras nas falas das idosas cuidadoras.

“Tem consequência positiva, porque pra mim é um presente de Deus ter deixado mamãe pra eu cuidar. Com ela eu aprendi mais o que é misericórdia, o que é ser humano e como nós somos frágeis." (Ent. 14)

"Eu acho que trouxe consequências positivas. Estou aprendendo mais. Nada de ruim vem, isso é um aprendizado pra gente." (Ent. 5) (...) consequência positiva, ah, eu acho que trouxe é muito amor, é bom, a gente aprende (...) (Ent. 13) 
"Só trouxe benefício, porque eu amo a minha irmã, eu tenho orgulho de cuidar dela e só eu que

(...) cuido. Tudo é gratificante para mim." (Ent. 6)

Os estudos de Araújo et. al. (2013) mostraram que as consequências positivas geralmente estão ligadas ao vínculo afetivo, conforme exemplificado na fala da entrevistada 6. Conforme mostrado neste estudo, Cruz et. al. (2010) também evidenciou que a satisfação advém do sentimento de dever cumprido, da preocupação manifestada pelos familiares para com o cuidador, da tranquilidade do idoso estar bem cuidado e dos laços afetivos. Em muitos momentos esse sentimento de dever cumprido se revela como um alívio para as cuidadoras.

\section{A sobrecarga da tarefa do cuidado - classe 3}

O cuidado familiar, ou seja, informal, tem sido a alternativa encontrada por muitas famílias para cuidar do seu idoso enfermo. Assim, a família se organiza, reúne e define estratégias de cuidado, elegendo um familiar que se responsabiliza pelas tarefas do cotidiano, surgindo, então, a figura do cuidador. Araújo et. al. (2013) definem como cuidador aquela pessoa que está mais próxima da pessoa idosa, cuidando e ajudando-a no dia a dia, seja nas atividades de vida diária, seja para atender aos cuidados prescritos por profissionais de saúde, conforme se observou neste estudo. E, pode ser a partir daí que surjam as difıculdades, limitações e sobrecarga física e emocional.

Ainda segundo os autores, o cuidar mostra-se como uma tarefa complexa, que deve atender às fragilidades do paciente e permitir a dinâmica e o conforto do lar. Dessa forma, cuidar não é uma tarefa fácil, pois exige uma mudança radical na vida de quem a executa, além de demandar atividades delicadas e sofridas (ARAÚJO et. al. 2013). Os relatos abaixo evidenciam essa realidade.

"Falei com meu filho que não estou aguentando mais, que vou colocar ele (esposo) no asilo. Está ruim, porque ele adoeceu e 
minha cabeça também atrapalhou toda." (Ent. 1)

"É que o serviço aumenta mais. O serviço acumulou por causa dele, tenho que ficar mais do lado dele." (Ent. 8)

"Me traz muita angústia, tem dia que estou muito brava com ele." (Ent. 18)

Conforme evidenciado nas entrevistas, cuidar acarreta sobrecarga e desconforto emocional à vida do cuidador, associada ao comprometimento das atividades da vida diária da pessoa idosa, revelando que a dependência dessa pessoa predispõe a sobrecarga no cuidador (GRATAO et. al. 2012). Desse modo, a rotina do cuidado pode se transformar em outra fonte de doenças para as mulheres idosas já acometidas pelas doenças crônicas degenerativas.

Além disso, ser cuidador exige renúncia pessoal e profissional. Araújo et. al. (2013) argumentam que o cuidador familiar sofre com alterações e abdicações em sua vida. Deste modo, tendem a distanciar-se da vida social à medida que a dependência da pessoa idosa se acentua. Essa realidade pode ser comprovada nas falas 03 e 14 descritas abaixo.

"Eu parei de trabalhar, mas eu tinha vontade de trabalhar fora por mais um tempo, igual eu trabalhava." (Ent. 03)

"Eu me anulei, me anulei em todos os sentidos, em todos." (Ent. 14)

Assim como demonstrado neste estudo, os cuidadores entrevistados no estudo de Jesus et. al. (2018) e Diogo et al. (2005) também relataram dificuldades por não ter vida social. Soma-se ainda a dificuldade de compartilhar os cuidados prestados, apesar das entrevistadas já terem relatado que recebem apoio de familiares, contudo, essa ajuda nem sempre é ofertada em um momento onde a necessidade se faz presente, se apresentam como apoio e quando há demanda de quem cuida. Tal realidade foi expressa pela entrevistada 16.

(...) Isso é difícil pra partilhar, por exemplo, se sou madrinha de um casamento e se não está no dia da minha irmã, infelizmente eu tenho que arranjar alguém de confiança para poder ficar." (Ent. 16) 
De acordo com Silva (2010, p. 141), cuidar de uma pessoa idosa pode ser um "empecilho que atrapalha o desenvolver 'normal' das atividades diárias de uma família, como passeios, programas de finais de semana etc.". Para Santana et al. (2012), geralmente há uma sobrecarga emocional e de atividades diárias que geram uma modificação na vida daquele que se propõe a assumir o papel de cuidador.

Todo esse contexto pode ser agravado quando as cuidadoras também são idosas, como no presente estudo, pois, além de todo trabalho no cuidado prestado, encaram as suas dificuldades e doenças, próprias do envelhecimento. Segundo Diogo et. al (2005), estas pessoas são denominadas "vítimas ocultas", uma vez que enfrentam o isolamento, a solidão e a sobrecarga de funções.

\section{Considerações finais}

Por meio deste estudo, pode-se concluir que o espaço relacional que envolve a pessoa idosa dependente e a mulher idosa cuidadora é diretamente afetado pelas mudanças que a demanda de cuidado proporcionam. As transformações repercutem no cotidiano não só da pessoa idosa cuidadora, mas de toda família, dos amigos e vizinhos e também da sociedade, que envolvidos com a situação, devem se tornar uma rede de apoio, possibilitando maior segurança e alívio para quem cuida.

Deste modo, a rede de apoio mais importante e presente no cotidiano das cuidadoras foi a dos filhos, amigos e vizinhos, ou seja, a rede de apoio informal. Em seguida, também foi relatada a rede de apoio formal, com a participação dos serviços da prefeitura, porém, os tipos de ajuda oferecida eram o fornecimento de medicamentos e agendamento de consultas em sua maioria, o que não contribui de modo efetivo nas atividades de cuidado executadas. A rede de apoio informal tem sido a mais frequente e atuante nos arranjos domiciliares em que há um idoso enfermo, o que pode ser explicado pela ausência e/ou ineficiência de ações da sociedade e do governo na promoção de meios que auxiliem o cuidador em sua função diária. 
A relação de cuidado é permeada por fatores positivos e negativos que recaem sobre a vida da idosa cuidadora. De um modo geral, as entrevistadas reconheceram como consequências positivas do cuidado a oportunidade de exercerem um ato de amor e retribuição, além de ser uma dádiva divina, ou seja, algo dado por Deus. Por outro lado, sobressaíram as consequências negativas do cuidado como o sentimento de "prisão", ou seja, o isolamento social, as renúncias pessoal e profissional e a mudança de rotina pelas quais se submetiam as mulheres. Além disso, um dos fatores negativos atrelados ao cuidado e presentes nesse estudo foi a sobrecarga física e emocional enfrentada pelas idosas, sobretudo, diante da sua velhice. Tomar conhecimento sobre as consequências negativas presentes na relação de cuidado possibilita compreender os potenciais riscos emocionais e físicos enfrentados pelos cuidadores, buscando a prevenção desses e a criação de estratégias que amenizem a sobrecarga e/ou que instrumentalize a vivência cotidiana dos mesmos.

Mediante tais resultados, torna-se evidente que a prestação de cuidados ao idoso merece destaque nas discussões políticas e sociais, assim como também no meio acadêmico, visando, sobretudo, o fornecimento de apoio, orientações e capacitações aos cuidadores familiares. Tal fato torna-se ainda mais importante e urgente quando se trata de idosas, executando uma atividade trabalhosa, informal e, em muitos casos, danosa a sua saúde. Assim, a criação de políticas de cuidado deve assistir o idoso debilitado, que devidamente atendido favorece o trabalho do cuidador, mas também, deve prestar assistência a quem cuida, principalmente, quando são mulheres a exercer esse papel, uma vez que essas tendem a vivenciar a sobrecarga de papéis em sua própria casa.

\section{Referências}

AEROSA, S. V. C., et al. Cuidar de si e do outro: estudo sobre os cuidadores de idosos. Psicologia, Saúde e Doenças, v. 15, n. 2, p. 482-494, 2014. 
ALMEIDA, A. V. O processo de feminização da velhice no município de Viçosa, MG: características, relações e risco social. 2015. 98 f. Dissertação (Mestrado em Economia Doméstica) - Universidade Federal de Viçosa, Viçosa-MG, 2015.

ALVES A. F. R., J. F. A. MONTEIRO. Repercussões Psicossociais na vida de cuidadores informais de pacientes acometidos por acidente vascular cerebral. Saúde e Transformação Social, v.6, n.3, p.26.-41, 2015. Disponível em:< https://www.redalyc.org/html/2653/265345667005/> Acesso em: 13 de Fev. 2019.

ANDRADE, F. M. M. O Cuidado Informal à Pessoa Idosa Dependente em Contexto Domiciliário: necessidades educativas do cuidador principal. 2009. Dissertação (Mestrado em Educação) - Instituto de Educação e Psicologia, Universidade do Minho, Portugal, 2009.

ARAÚJO, J. F., et. al. Perfil dos cuidadores e as dificuldades enfrentadas no cuidado ao idoso, em Ananindeua, PA. Rev. Bras. Geriatr. Gerontol., Rio de Janeiro, v. 16, n.1, p.149-158, 2013.

BIERNACKI, P.; WALDORF, D. Snowball sampling: problems and techniques of chain referral sampling. Sociological Methods \& Research, Thousand Oaks, CA, v. 10, n. 2, 1981.

BIOLO, H. F., PORTELLA, M. R. Vivência do cuidador familiar: casos acompanhados pela estratégia da saúde da família de Passo Fundo-RS. Estud. Interdiscipl. Envelhec., v. 15, n. 2, p. 177-195, 2010.

CAMARGO, B. V.; JUSTO, A. M. IRAMUTEQ: Um software gratuito para análise de dados textuais. Temas em Psicologia, Ribeirão Preto, v. 21, n. 2, p. 513-518, dez. 2013. Disponível em: <http://pepsic.bvsalud.org/scielo.php?script=sci_arttext\&pid=S1413-389X2013000200016\&lng=pt\&nrm=iso>. Acesso em: 10 de Fev. 2019.

CRUZ, D. T.; CAETANO, V.C.; LEITE, I.C.C. Envelhecimento populacional e bases legais da atenção à saúde do idoso. Cad. Saúde Colet., Rio de Janeiro, v. 18, n. 4, 2010.

DÁ MESQUITA, J. A. R. Suporte social e redes de apoio social em idosos. 2011. 27 f. Dissertação (Mestrado em Psicologia Clínica) - Instituto Universitário, Lisboa, Portugal, 2011.

DIOGO, M. J. D., et al. Orientações para idosas que cuidam de idosos no domicílio. Rev Esc Enferm USP, v.39, n. 1, p.97-102, 2005. 
DOMINCUES, M. A., et al. Rede de suporte social de idosos do Programa Universidade Aberta à Terceira Idade da Escola de Artes, Ciências e Humanidades da Universidade de São Paulo. Rev Kairos. v.15, n.7, p.33-51, 2012.

GRATÃO, A. C. M., et al. Sobrecarga e desconforto emocional em cuidadores de idosos. Texto Contexto Enferm, Florianópolis, v.21, n.2, p.304-12, 2012.

GUEDES, S. I. C. M. Cuidar de idosos com dependência em contexto domiciliário: necessidades formativas dos familiares cuidadores. 2011. $117 \mathrm{f}$. Dissertação (Mestrado em Enfermagem Comunitária) - Escola Superior de Enfermagem do Porto, Portugal, 2011.

IBCE - Instituto Brasileiro de Geografia e Estatística. Censo demográfico 2010. Disponivel em: < http://www.ibge.gov.br/home/estatistica/populacao/censo2010/default.shtm>. Acesso em: 09 de Fev. 2019.

JESUS, I. T. M. de., et al. Sobrecarga, perfil e cuidado: cuidadores de idosos em vulnerabilidade social. Rev. Bras. Geriatr. Gerontol., v.21, n. 2, p. 199-209, 2018.

MAYOR, M. S., RIBEIRO, O., PAUL, C. Satisfaction in dementia and stroke caregivers: a comparative study. Rev. Latino- Am. Enfermagem, v. 17, n. 5, p. 620-624. Disponível em:<http://www.scielo.br/pdf/rlae/v17n5/04.pdf> Acesso em: 10 de Fev. 2019

MAZZA, M. P. R. O cuidado em família sob o olhar do idoso. 2008. 178 f. Tese (Doutorado em Saúde Pública) - Faculdade de Saúde Pública da Universidade de São Paulo, São Paulo, 2008.

MINISTÉRIO DA SAÚDE/DATASUS, 2015. População residente - Estudo de Estimativas Populacionais por Município, Idade e Sexo 2000-2015 - BRASIL. Disponivel em:< http://tabnet.datasus.gov.br/cgi/deftohtm.exe?novapop/cnv/popbr.def > Acesso em: 7 de Fev. 2019.

MIRANDA, L. C. V. Fatores associados à qualidade de vida de idosos de um Centro de Referência, em Belo Horizonte, Minas Gerais. 2014, 114f. Dissertação (Mestrado em Enfermagem). Universidade Federal de Minas Gerais, Belo Horizonte. 2014. Disponivel em:<http://www.enf.ufmg.br/pos/defesas/839M.PDF> Acesso em: 10 de Fev. 2019.

SANTANA, S. F., et al. Indicativos de aplicabilidade das orientações de enfermagem no cotidiano de cuidadores de portadores de Alzheimer. Rev EsC Enferm USP, v. 43, n. 2, p.459-464, 2009. 
SILVA, V. da. Qualidade de vida do idoso: cuidado do idoso, dever de quem? Revista Espaço Acadêmico, n. 110, 2010.

SOUZA, J; KANTORSKI, L. P; MIELKE, F. B. Vínculos e redes sociais de indivíduos dependentes de substâncias psicoativas sob tratamento em CAPS AD. SMAD, Rev Eletrônica Saúde Mental Álcool e Drogas. 2006. v. 2 (1):1-17. Disponível em:<http://pepsic.bvsalud.org/pdf/smad/v2n1/v2n1a03.pdf>. Acesso em: 10 de Fev. 2019.

\section{Notas}

1 Este artigo apresenta os resultados parciais do doutorado concluído no Programa de Pós-Graduação em Economia Doméstica da Universidade Federal de Viçosa (UFV), MG, no ano de 2019.

2 Bacharel em Economia Doméstica pela Universidade Federal de Viçosa (UFV), Mestre e Doutora pelo Programa de Pós-Graduação em Economia Doméstica pela UFV. Brasil. ORCID: 0000-0003-0210-9117. E-mail: avaalessandra@yahoo. com.br

3 Doutora em Engenharia de Produção pela Universidade Federal de Santa Cata-rina (UFSC). Docente do Departamento de Economia Doméstica e do Programa de Pós-Graduação em Economia Doméstica da Universidade Federal de Viçosa (UFV). Brasil. ORCID: 0000-0003-2247-2327. E-mail: sctmafra@ufv.br

4 Doutora em Ciência Florestal pela Universidade Federal de Viçosa (UFV). Professora da Faculdade Dinâmica do Vale do Piranga. Brasil. ORCID: 0000-00018130-5196. E-mail: emiliapiosilva@yahoo.com.br

5 Doutora em Saúde Pública pela Escola Nacional de Saúde Pública (ENSP/Fiocruz). Brasil. ORCID: 0000-0001-7044-7754. E-mail: solange.kanso@gmail.com

6 Doutora em Antropologia Social pela Universidade de São Paulo (USP). Docen-te do Departamento de Extensão Rural e do Programa de Pós-Graduação em Extensão Rural da Universidade Federal de Viçosa (UFV). Brasil. ORCID: 00000003-0310-9055. E-mail: sheiladoula@gmail.com.

7 Estimativa elaborada pelo Ministério da Saúde/ SVS/CGIAES. Disponível pelo link: http://tabnet. http://tabnet.datasus.gov.br/cgi/deftohtm.exe?novapop/cnv/ popbr.def. Acesso em 17 de fevereiro de 2019. 


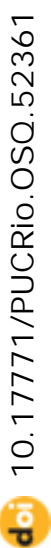

